\title{
National Criticality Experiments Research Center (NCERC) - capabilities and recent measurements
}

\author{
Nicholas Thompson $^{1 *}$, Jesson Hutchinson ${ }^{1}$, Rian Bahran ${ }^{1}$, David Hayes ${ }^{1}$, William Myers ${ }^{1}$, Jennifer Arthur ${ }^{1}$, John \\ Bounds $^{1}$, Theresa Cutler ${ }^{1}$, Derek Dinwiddie ${ }^{1}$, Joetta Goda ${ }^{1}$, Travis Grove ${ }^{1}$, Robert Little ${ }^{1}$, George McKenzie ${ }^{1}$, Alex \\ McSpaden $^{1}$, Rene Sanchez ${ }^{1}$, Avneet Sood ${ }^{1}$, Morgan White ${ }^{1}$, and Robert Margevicius ${ }^{1}$
}

${ }^{1}$ Los Alamos National Laboratory, P.O. Box 1663, Los Alamos, NM 87545

\begin{abstract}
The National Criticality Experiments Research Center (NCERC) located at the Device Assembly Facility (DAF) at the Nevada National Security Site (NNSS) and operated by Los Alamos National Laboratory (LANL) is home to four critical assemblies which are used to support of range of missions, including nuclear criticality safety and nuclear nonproliferation. Additionally, subcritical systems can also be assembled at NCERC. NCERC is providing critical and subcritical experiments valuable to the nuclear data community and experiments performed at NCERC are often published as benchmarks in the International Criticality Safety Benchmark Evaluation Project (ICSBEP) Handbook. This manuscript will give a broad overview of recent experiments performed at NCERC, upcoming experiments, and why integral measurements are important and useful to the nuclear data community.

The four critical assemblies are GODIVA IV, FLATTOP, COMET, and PLANET. GODIVA IV is a cylindrical metal fast burst reactor, the fourth in the GODIVA series that dates back to the 1950's. FLATTOP is an highly enriched uranium (HEU) or Pu core reflected by natural uranium. COMET and PLANET are vertical lift assemblies, where one half of the reactor can be lifted to the upper half of the reactor to create a critical system. Some recent experiments include various critical intermediate energy assemblies with lead, and subcritical measurements of plutonium reflected by copper, tungsten, and nickel. Work is also underway to make a better measurement of the critical mass of neptunium, using a neptunium sphere surrounded by nickel shells. Additionally, measurements will be performed next year with HEU shells from Rocky Flats. These HEU shells will be stacked together to make larger systems, allowing for a large range of criticality (from subcritical to delayed critical). Other upcoming measurements include an HEU critical assembly sensitive to intermediate energy neutrons.
\end{abstract}

\section{Introduction}

Integral experiments are vitally important to many areas of nuclear engineering. Criticality safety engineers use the data from integral experiments to refine reactivity estimates and calculate bias in their results [1]. Nuclear data experts use integral experiments to validate the quality of nuclear data evaluations and libraries. Nuclear reactor designers use the data from integral experiments to help in the design, analysis, and uncertainty quantification of their systems.

The National Criticality Experiments Research Center (NCERC) is the only general purpose critical experiments facility in the US, and is one of only a few left in the world. At NCERC, subcritical, critical, and above prompt critical measurements are made, using four critical assemblies and other Radiation Test Objects (RTO). This paper will detail some of the capabilities at NCERC, and some of the recent measurements made there.

\section{NCERC}

NCERC is operated by Los Alamos National Laboratory (LANL), and is located at the Device Assembly Facility (DAF) at the Nevada National Security Site (NNSS), previously known as the Nevada Test Site. LANL operated critical experiments in Los Alamos from 1946-2004 (then known as the Critical Experiments Facility); after 2004, the critical assemblies and materials were relocated to the DAF at NNSS.

Today, NCERC supports many US Government programs and missions, as well as many US and international collaborations. Recent collaborators include many US universities, private companies, and AWE in the UK, IRSN and CEA in France, and the JAEA in Japan.

Measurements performed at NCERC are regularly documented and published as critical benchmarks for the International Criticality Safety Benchmark Evaluation Project Handbook (ICSBEP) [2], allowing international use of these important data. 


\subsection{Critical Assemblies}

NCERC has four critical assemblies, Comet, Planet, FlatTop, and Godiva IV. Each is unique and suited for different measurements, allowing for measurements of a wide range of materials, reactivities, and neutron energy spectra.

Comet (shown in Figure 1) and Planet (shown in Figure 2) are a general purpose vertical lift assemblies. A vertical lift assembly is a machine where the core of the reactor/measurement is split into two halves, with half the fuel/material on the top, immovable plate, and half the fuel/material on the bottom, a movable platen. To bring the system critical, the bottom plate is lifted towards the top plate, and as the two halves of the core come closer together, reactivity increases. If there is enough fuel, the system can be brought to a critical state.

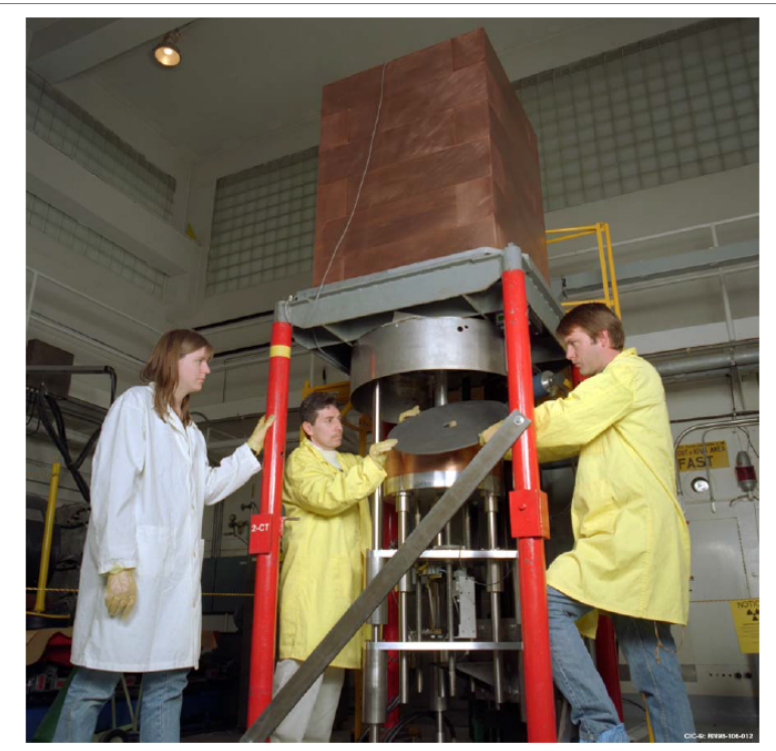

Figure 1: Comet critical assembly.

For both Comet and Planet, in normal measurements, layers of fuel and other materials are stacked in order to make various critical or near critical configurations. Possible fuel layers include the high enriched uranium (HEU) Jemima plates (so named because they resemble pancakes), HEU class foils, natural uranium plates, and plutonium plates from the Zero Power Physics Reactor (ZPPR) machine. These fuel plates can be mixed with each other and with other materials (for example, moderating materials to slow down neutrons, or other materials of interest). Planet is a smaller machine, with a lower maximum weight that can be lifted. Comet is the 'heavy duty' machine, and is better suited for intermediate neutron energy measurements where large amounts of material and reflectors are often necessary.

Flat-Top (shown in Figure 3) is an assembly where various spherical cores of special nuclear material (HEU or plutonium) can be reflected by a large ( 0.48 meter od) spherical natural uranium reflector. This spherical reflector is separated in three pieces - a stationary hemisphere, and two movable quarter spheres on tracks. When the as-

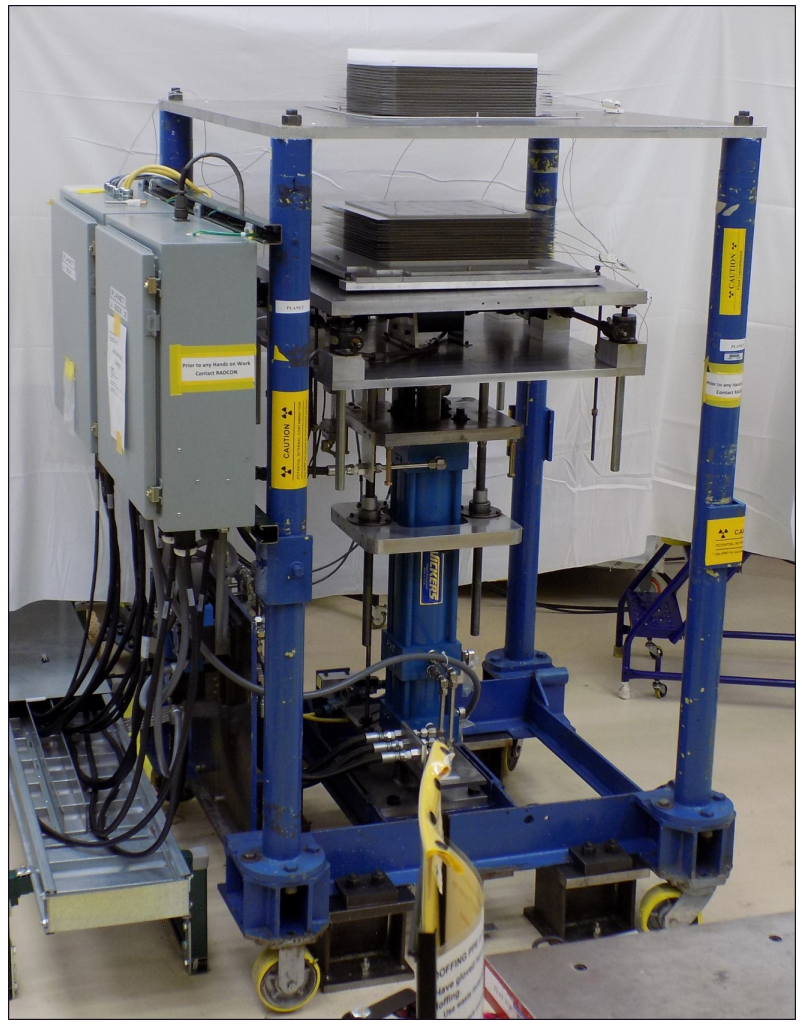

Figure 2: Planet critical assembly.

sembly is not operating, the two quarter spheres are separated from the stationary hemisphere to keep the system very subcritical and allow work to be done. During operation, the two quarter spheres are slowly moved closer to the stationary assembly until the system is fully 'closed', where all three pieces are touching and combined make a spherical reflector. For fine control of reactivity, three control rods are present in the stationary hemisphere and can be raised and lowered from beneath the assembly. These control rods are also made of natural uranium - inserting them in the core increases reactivity and pulling them out of the core decreases reactivity. Samples can be placed inside small holes in the core or the reflector, depending on the measurement.

The last critical assembly is Godiva IV (shown in Figure 4). Godiva IV is a fast burst assembly, and can be operated at reactivities above prompt critical. It is made up of five cylindrical rings of $\mathrm{HEU}\left(93.5 \% \mathrm{w} / \mathrm{o}^{235} \mathrm{U}\right)$ with $1.5 \% \mathrm{Mo}$, and reactivity is controlled with a number of control rods, a safety block, and a burst rod (all made of HEU). The shortest fast burst that can be achieved is 24 $\mu$ s FWHM, with a maximum power of $90 \mathrm{GW}$.

Subcritical systems can also be assembled at NCERC. In the past five years, about 30 subcritical systems measured at NCERC have been benchmarked, some photos from these measurements are reproduced below as Figure 5. These systems normally have a spherical core of special nuclear material surrounded by different reflectors and moderators. One such core, an $\alpha$-phase plutonium core called the BeRP ball, has been measured where it is surrounded by materials such as nickel, tungsten, copper, 


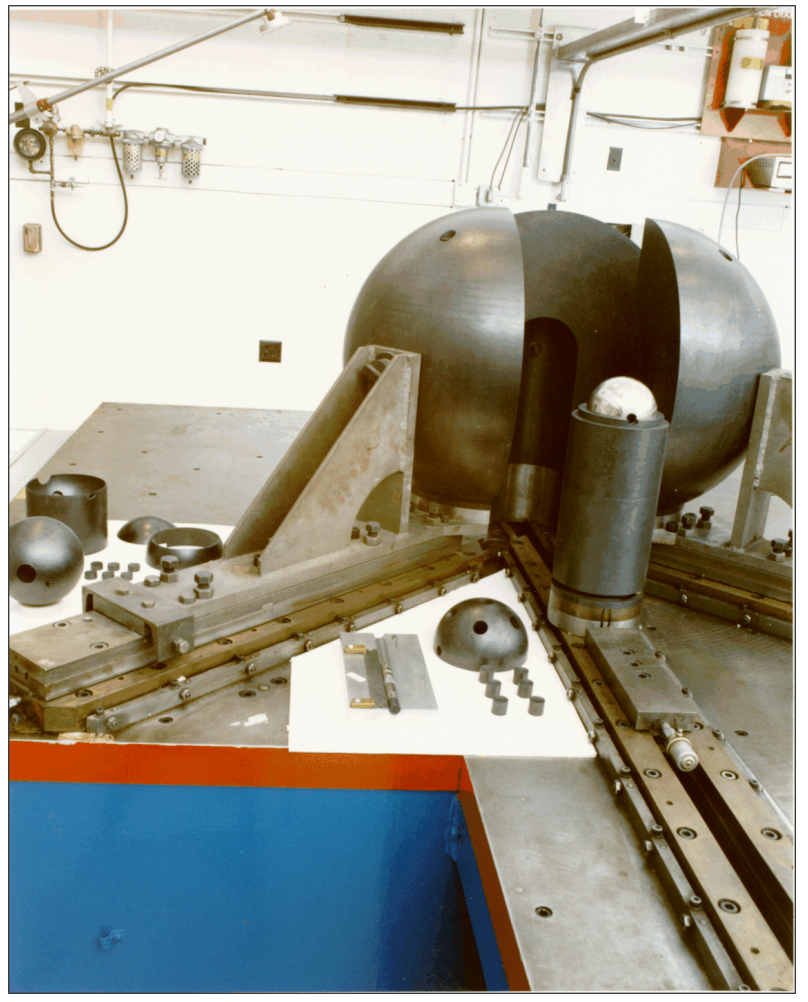

Figure 3: Flat-Top critical assembly.

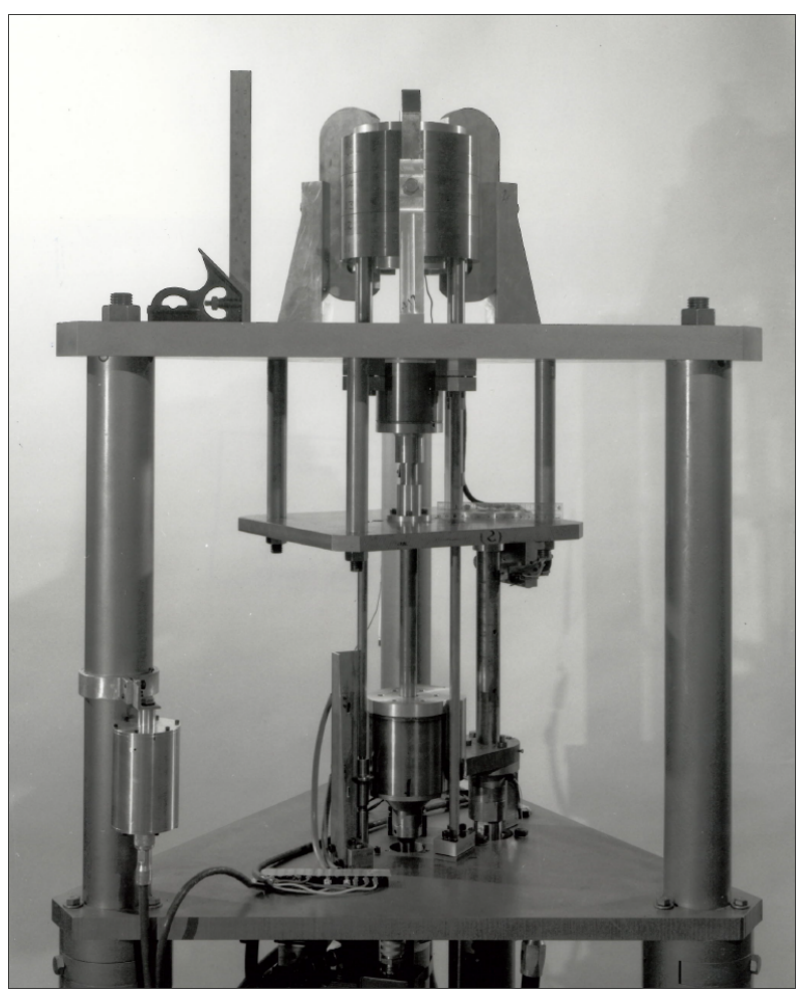

Figure 4: Godiva IV fast burst assembly.

and polyethylene. Surrounding the assembly in these measurements are large detector systems known as NoMAD detectors - these detectors have $15 \mathrm{He}-3$ tubes each, and measure count rates and detections in coincidence. These measurements are particularly sensitive to $\bar{v}$ (average number of neutrons per fission) and the distribution of $v$ [3].

Experiments are typically designed using computational tools such as $\mathrm{MCNP}^{\circledR 1}$ [4]. Of particular importance when designing experiments is the neutron energy spectrum and the k-eff sensitivity for materials of interest; these values can be computationally calculated using MCNP and other simulation tools. In many cases, sensitivity for a specific material/element/isotope is being maximized for a specific target energy range, while also maintaining a k-eff of 1.0 so that the system will be critical.

After experiments are performed, they are documented, published, and written up as ICSBEP benchmarks. The ICSBEP working group reviews the potential new benchmarks in a thorough review process, and if the experiment is documented well, it's accepted as a benchmark where scientists and researchers all over the world can use this data and these models to determine the quality of nuclear data, validate nuclear simulation codes, and determine potential performance of new systems being designed.

\section{Recent and Upcoming Measurements}

\subsection{NCERC/JAEA Measurements}

NCERC, in collaboration with the Japan Atomic Energy Agency (JAEA), recently measured performed multiple critical measurements with lead $(\mathrm{Pb})$. The JAEA was interested in making measurements related to lead-bismuth cooled accelerator driven systems (ADS). Three series of measurements were performed, one with $\mathrm{Pu}$, one with HEU, and one with "LEU".

In the plutonium measurement series, plutonium plates from the ZPPR experiment (3" x 2" x 0.25 ") were placed in aluminum boxes with plates of lead, as can be seen in Figure 6 and Figure 7 [5][6]. To simulate a hypothetical void in the lead-bismuth coolant of an ADS, measurements were also made where some of the lead plates were replaced with aluminum fillers. The core was also surrounded by a copper reflector, resulting in the system having an intermediate neutron energy spectrum.

The HEU measurements used the "Jemima" plates ( 21 $\mathrm{kg}$ of HEU) interleaved with plates of lead (see Figure 8) [7]. Unlike the plutonium plates, which are more shaped like credit cards, the Jemima plates are very flat cylinders, resembling large pancakes. These plates were stacked with plates of lead, with roughly half of the core on the top resting on the stationary stainless steel diaphragm, and half the core resting on the lower moveable platen. These measurements also used a large copper reflector to keep the system in the intermediate neutron energy spectrum.

The "LEU" measurements were very similar to the HEU series, with one crucial difference. HEU plates were

${ }^{1} \mathrm{MCNP}^{\circledR}$ and Monte Carlo N-Particle ${ }^{\circledR}$ are registered trademarks owned by Triad National Security, LLC., manager and operator of Los Alamos National Laboratory. Any third party use of such registered marks should be properly attributed to Triad National Security, LLC., including the used of the designation as appropriate. For the purposes of visual clarity, the registered trademark symbol is assumed for all references to MCNP within the remainder of this paper. 


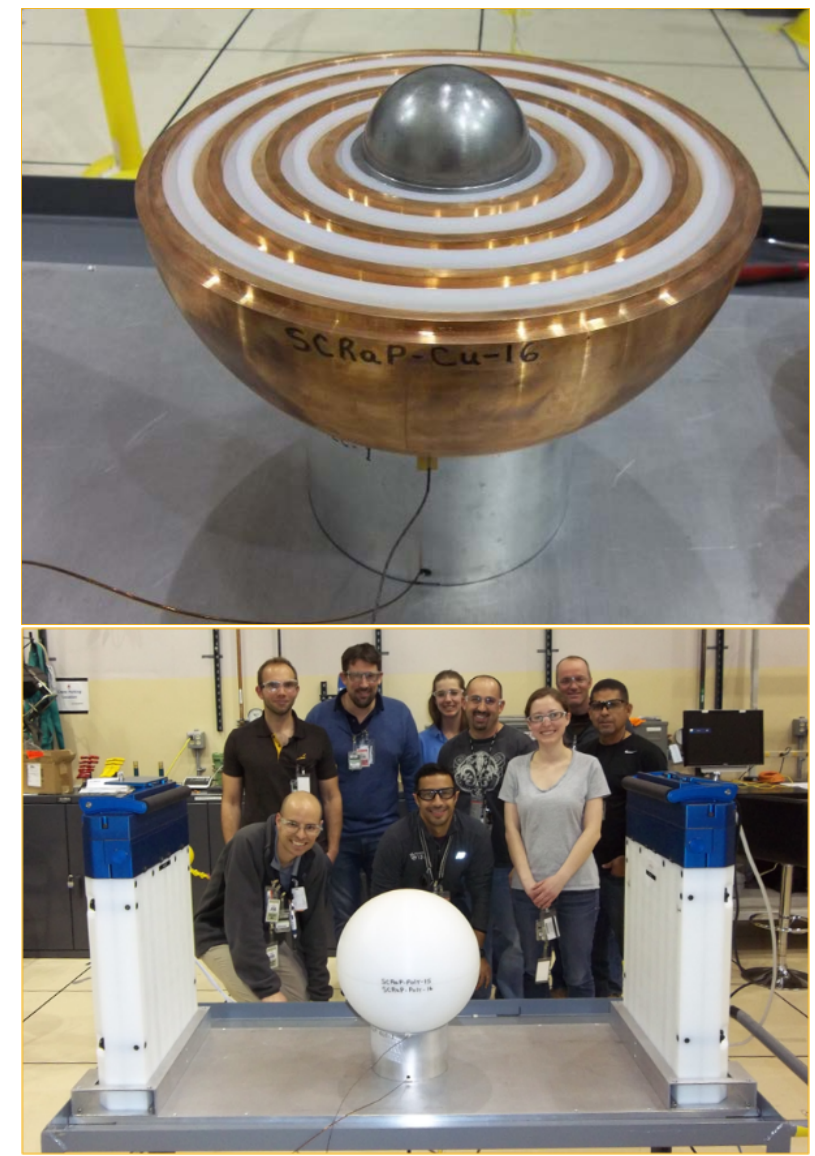

Figure 5: Measurements of subcritical assemblies at NCERC.

used, but there were also many plates of natural uranium. The "effective enrichment" of the fuel, adding the HEU and natural $U$ plates together was about $21 \%$, very similar to the high assay low enriched uranium (HALEU) fuel currently be investigated for use in advanced reactors and micro-reactors. All three of these series of measurements will be submitted to the ICSBEP in the next two years

\subsection{Recent Subcritical Measurements}

As mentioned above, the BeRP ball (an $\alpha$-phase spherical $\mathrm{Pu}$ core) has been used in numerous subcritical measurements recently. In 2012, the BeRP-Ni measurement series was executed, where the BeRP ball was reflected by varying thicknesses of nickel by adding shells of nickel around the core. These measurements were published in the ICSBEP in 2014. The next set of measurements were the BeRP-W, where instead of nickel shells, the shells were made of Tungsten: these measurements were published in 2016. Most recently were the SCR $\alpha \mathrm{P}$ measurements, which used copper and polyethylene shells, with different numbers and mixtures of shells in the different configurations: these measurements were published in 2018 [8][9].

\subsection{Upcoming Measurements}

Many measurements are currently in the planning process, one of these measurements is called $\mathrm{NeSO}$, or Neptunium

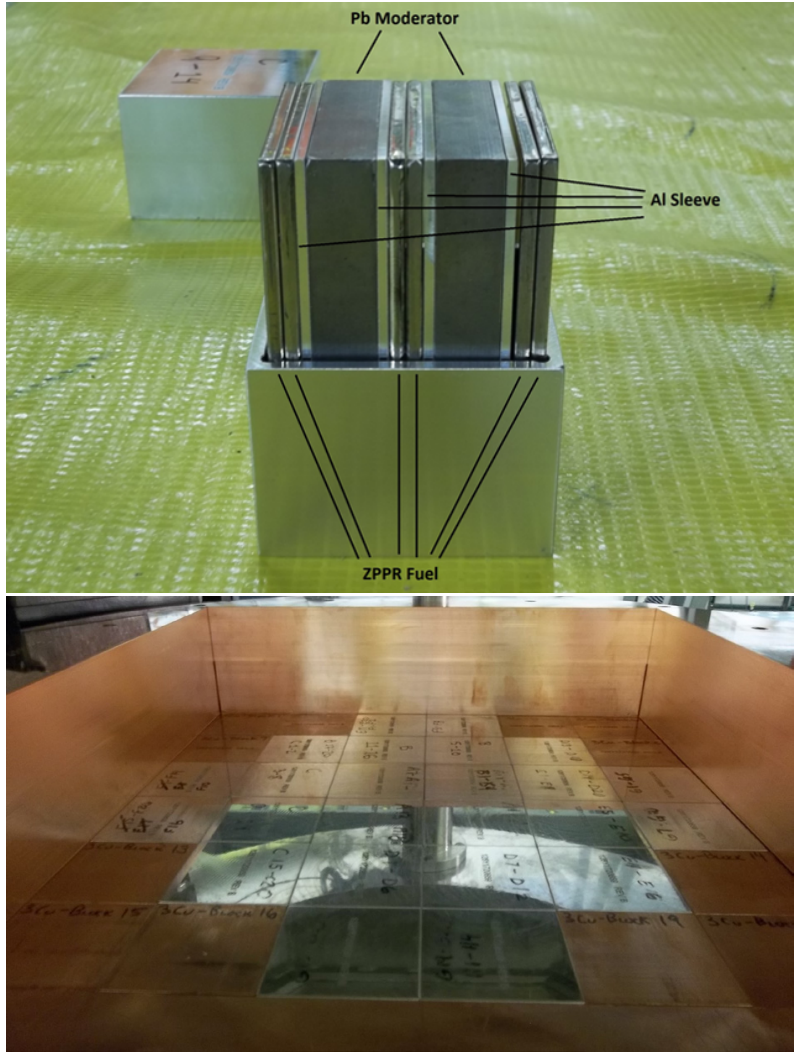

Figure 6: Lead plutonium measurements.

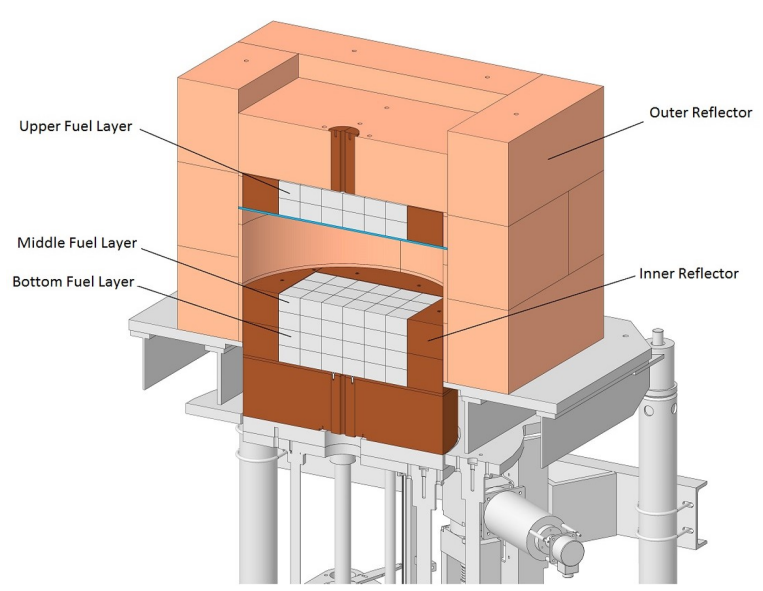

Figure 7: Lead plutonium measurements, visualization of the full assembly.

Subcritical Observation [10]. During the NeSO experimental series, a spherical neptunium core will be reflected by various reflectors (shown in Figure 9), partially to get a better estimate of the critical mass of neptunium, and partially to test the quality of nuclear data parameters for neptunium such as $\bar{v}$.

Another measurement series will use the Rocky Flats shells, HEU nesting shells of a large range of sizes (shown in Figure 10) which will be used to create subcritical and critical configurations. In a similar vein, the CURIE ex- 


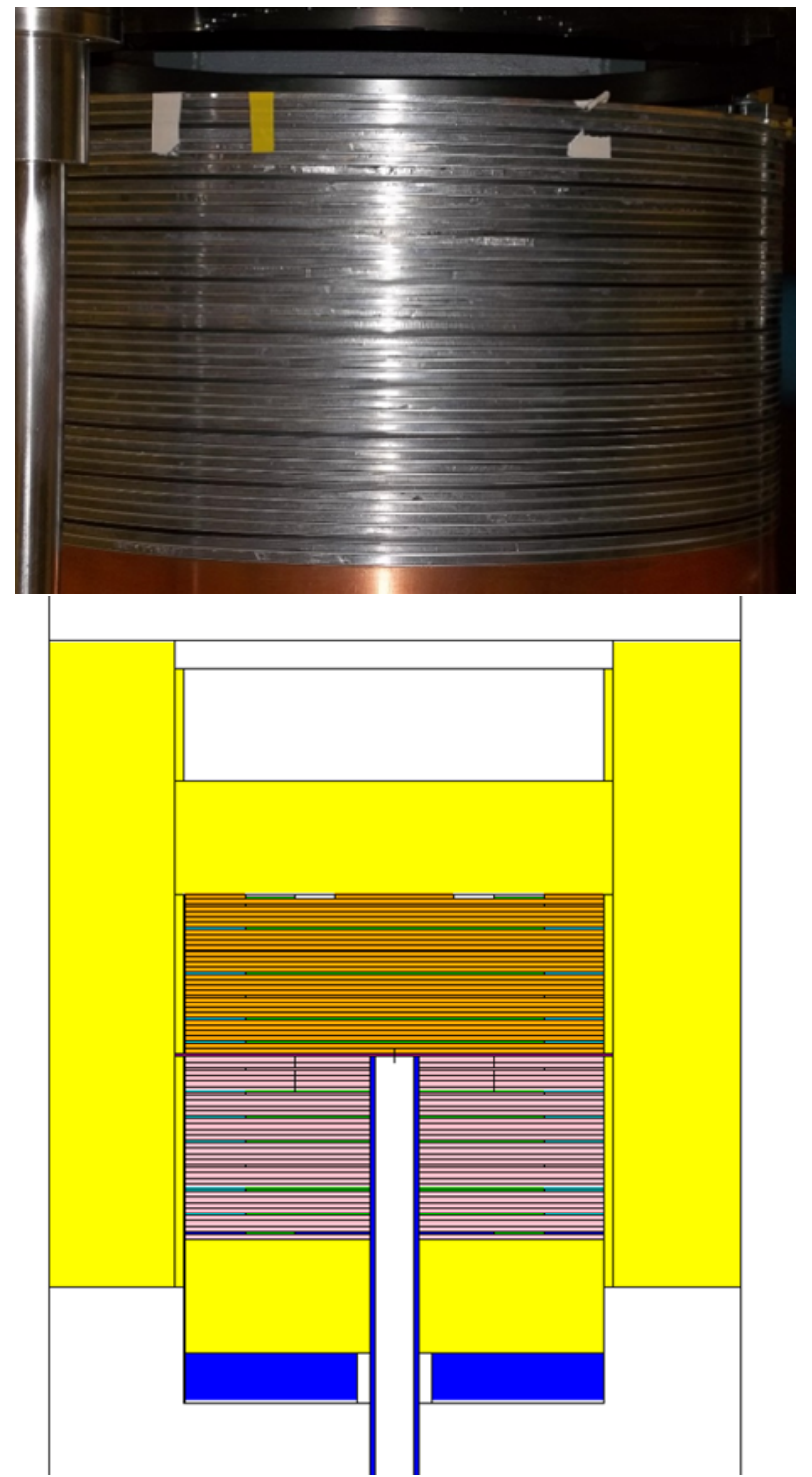

Figure 8: Jemima HEU plates and lead plates on Comet, photo and cross sectional view.

periment series will use the Comet assembly and the HEU "Jemima" plates layered with Teflon to create intermediate energy critical systems for testing the quality of ${ }^{235} \mathrm{U}$ nuclear data in the unresolved resonance region.

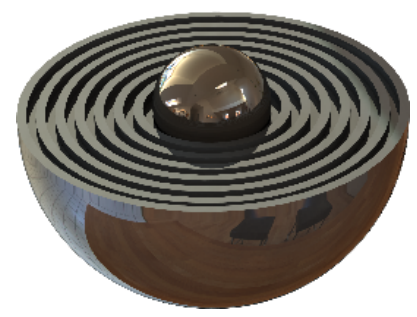

Figure 9: Visualization of the NeSO experiment.

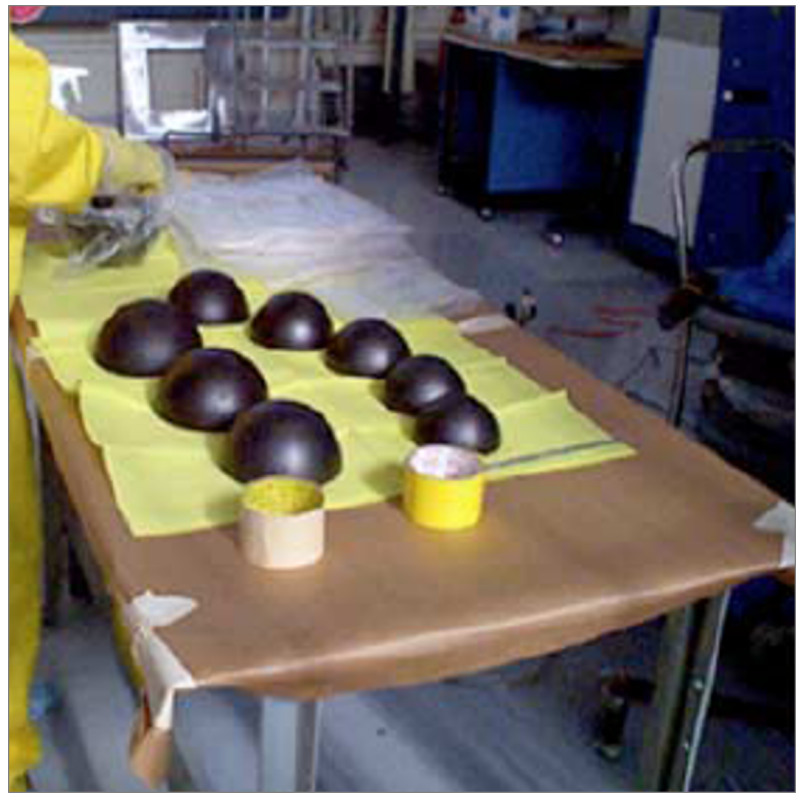

Figure 10: Rocky Flats HEU nesting shells.

\section{Acknowledgments}

NCERC is supported by the DOE Nuclear Criticality Safety Program, funded and managed by the National Nuclear Security Administration for the Department of Energy.

\section{References}

[1] B. Kiedrowski et al., "Whisper: Sensitivity/Uncertainty-Based Computational Methods and Software for Determining Baseline Upper Subcritical Limits." Nuclear Science and Engineering 181:1, 17-47 (2015).

[2] J. Bess, T. Ivanova, "International Handbook of Evaluated Criticality Safety Benchmark Experiments," NEA1486/16. Nuclear Energy Agency, Paris, September 2018 Edition.

[3] J. Arthur et al., "Validating the performance of correlated fission multiplicity implementation in radiation transport codes with subcritical neutron multiplication benchmark experiments." Annals of Nuclear Energy. 120, 348-366 (2018).

[4] C.J. WERNER, et al., "MCNP6.2 Release Notes", Los Alamos National Laboratory, LA-UR-18-20808 (2018).

[5] J. Goda et al., "Comparison of Methods for Determining Multiplication in Subcritical Configurations of a Plutonium System." Proceedings of the PHYSOR 2018. Cancun, Mexico (2018).

[6] G. McKenzie, "Prompt Neutron Decay Constant Measurements on a Lead Moderated, Copper Reflected Weapons Grade Plutonium System." Proceedings of the Advances in Nuclear Nonproliferation Technology and Policy Conference. (2018).

[7] M. Fukushima et al., "Lead Void Reactivity Worth in Two Critical Assembly Cores with Differing Uranium 
Enrichments." Nuclear Science and Engineering 189:1, 93-99 (2017).

[8] J. Hutchinson et al., "Subcritical Copper Reflected $\alpha$ phase Plutonium (SCR $\alpha \mathrm{P})$ Measurements and Simulations." Proceedings of M\&C 2017 - International Conference on Mathematics \& Computational Methods Applied to Nuclear Science \& Engineering. Jeju, Korea, (2017).
[9] J. Hutchinson et al., "Measurements on a Subcritical Copper-Reflected $\alpha$-phase Plutonium (SCR $\alpha \mathrm{P})$ Sphere." Transactions of the American Nuclear Society. Washington, D.C. 117, 832-835 (2017)

[10] R. Bahran et al., "Neptunium Subcritical Observation (NeSO) Integral Benchmark Experiment Design." Transactions of the American Nuclear Society. Washington, D.C. 117, 853-856 (2017) 